\title{
Two-dimensional code security image and its application in design of food traceability system
}

\author{
Feng Hui, Luo Bin \\ Faculty of Mechanical Electronic and Information, Jiangsu Polytechnic of finance \&Economics, \\ Kengaraga No.8, MeiCheng East Road, HuaiAn, China \\ hunter2011@foxmail.com
}

\begin{abstract}
Keywords: Two-dimensional code image; food safety; transaction monitoring terminal; Food traceability; logistics management.
\end{abstract}

\begin{abstract}
Two-dimensional code technology has been applied to all aspects of production and life, such as auto parts production line tracking, product traceability, medical emergencies, e-commerce, media and tourism. However, these two-dimensional code application generally concentrated in record numbers, letters and words, but in practice many applications need to record the image, such as the admission of students who require a printed picture as a security basis. Establish an efficient food traceability systems available, one aspect of the need to analyze the food production and processing sectors in the more complex and require the use of efficient and reliable two-dimensional code identification and data collection related equipment; on the other hand to analyze the correlation algorithm for large data storage and traceability information model.
\end{abstract}

\section{Introduction}

In recent years, food safety issues widespread concern in the community and public opinion, it is related to people's health and life safety of the livelihood of people [1]. The sales cycle data collection, storage, make the appropriate inquiries and supervision and management, and provide consumers with accurate, efficient and credible food information platform, the establishment of information traceability system is an ideal tool for effective management of food safety [2].

In the construction of traceability system, involving every aspect of food production, transportation, storage, etc. should be able to be recorded, it can be traced back $[3,4]$. Food needs to be monitored by IT to connect through the appropriate bar code reading device and the internet, to achieve effective management of food information [5]. When it comes to research and development in food traceability system, how to integrate business data really a industry chain, establish and improve data collection, transmission, storage, query system model, multi-user, multi-sectoral inquiry, supervision, traceability and other functions, is reform should focus on system design issues need to be developed considering the time [6]. To sum up, really want to establish a food traceability regulatory decision-making platform, making the government decision-making and food control data can be effectively supported and technical support from the platform, forming a food traceability system based on information technology [7].

The study of two-dimensional code image storage and identification has important significance. This paper presents a two-dimensional code technology for product traceability applications logistics system solutions through the use of information technology. Establish and improve the food safety traceability system for food production, circulation can be traced back to do, you can query, manageable, of which the origin of the raw materials for the food information, logistics information.

\section{Related theory of Two-dimensional code image}

Traceability security information management. According to statistics, at present our products in the circulation costs account for $40 \%$ to $60 \%$ of the total cost, which not only increases the burden on consumers, but also seriously affected the promotion of their products. Production-oriented enterprises through the establishment of the logistics management system, the products in circulation dropped 20 percent less cost. Therefore, the establishment of information security products, logistics 
control system is very important.

For the above, the system gives business every product a unique set of logistics and security monitoring identified in accordance with international rules for the preparation of coding. Enterprises can be printed as password security logo pasted on the product. Consumers can call the National Unity Enquiries, enter the security code on the product, check the authenticity of particular products, understand the product and business related information. Its logistics product security information management system flow was shown in Fig. 1.

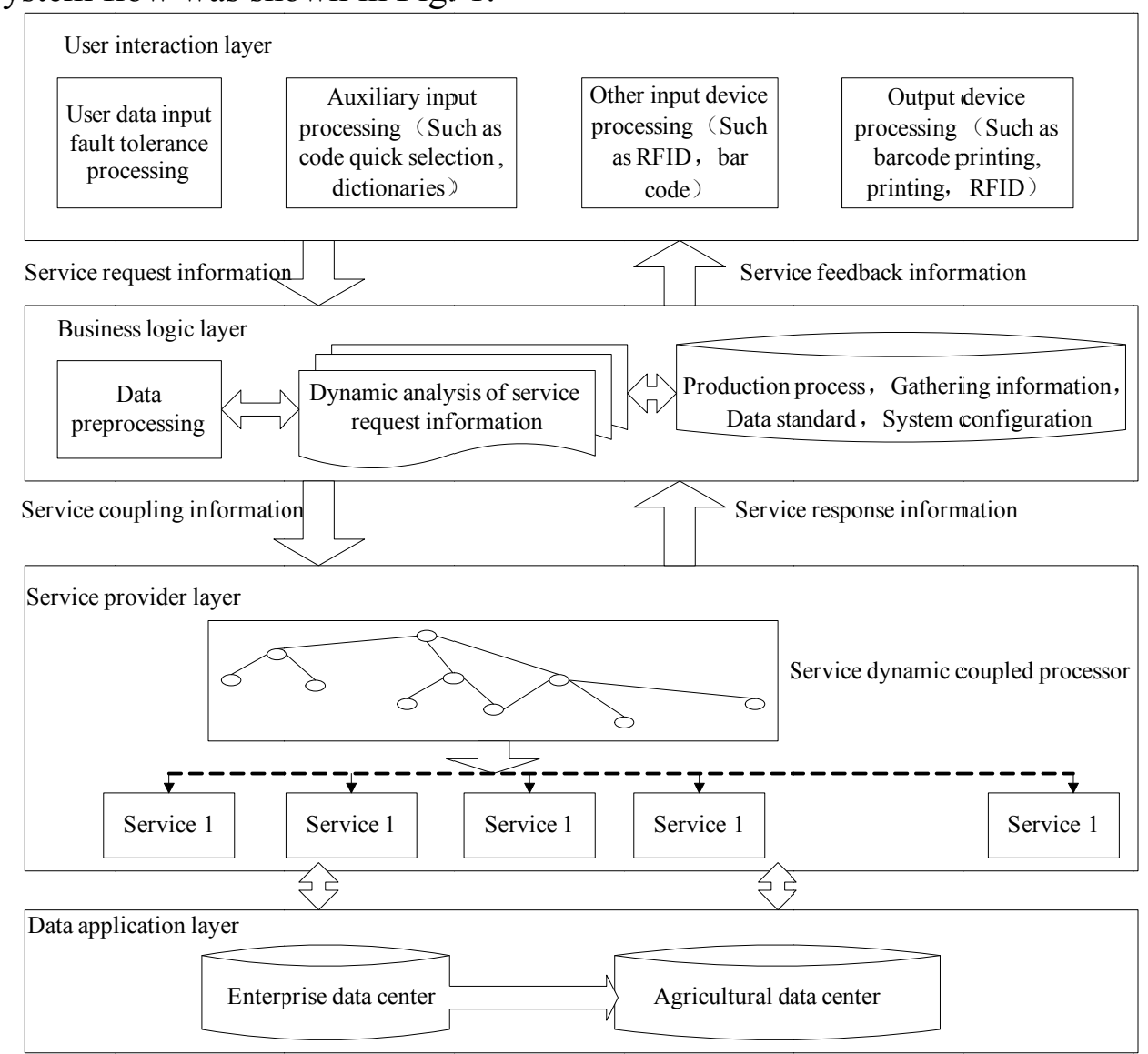

Fig. 1. Logistics security system flowchart

Dimensional bar code classification. A two-dimensional bar code information capacity, a wide range of coding, security is good and strong error correction capability, these features fit in the information on commodity tracking, search, forms, and other aspects of confidentiality.

The test uses two monochrome CCD industrial cameras, determine the coordinates of the location of the left and right of the camera, the camera center distance of about $15 \mathrm{~cm}, 10 \mathrm{~cm} \sim 20 \mathrm{~cm}$ logistics products for the distance from the camera, the speed detection platform is $5 \mathrm{~cm} / \mathrm{s}$, each of logistics products different packaging, different anti-counterfeit labels image position, under the above experimental conditions tested. Logistics Product security two-dimensional code image was shown in Fig. 2.

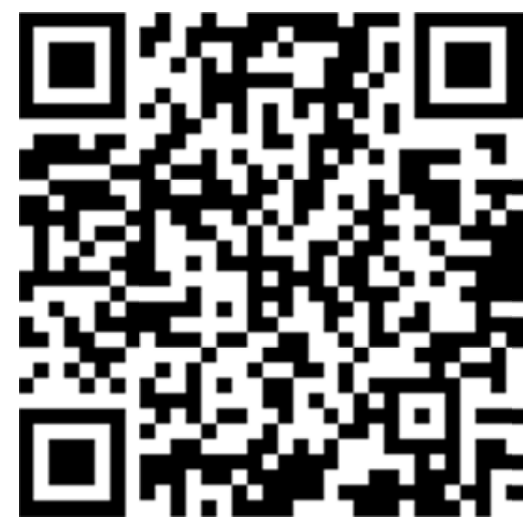

Fig. 2. Logistics Product security two-dimensional code image 
Quantum behaved particle swarm optimization-Support Vector Machine and Its Optimization. Support vector machine (SVM) is a new and promising technique for data regression and classification. In this section a brief description of SVM is given. Assume $\{(\mathrm{x} 1, \mathrm{y} 1), \ldots(\mathrm{xl}, \mathrm{yl})\}$ be the given training data sets, where each $x_{1} \subset R^{n}$ shows the input space of the sample and has a corresponding target value $y_{1} \subset R$ for $i=1 \cdots|\mathrm{i}|$ where represents the size of the training data. The support vector regression solves an optimization problem:

$$
\frac{1}{2}\|\mathrm{w}\|^{2}+C \sum_{i=0}^{l}\left(\xi+\xi^{*}\right)
$$

where ${ }^{X_{i}}$ is mapped to a higher dimensional space by the function $f,{ }_{i}$ is the upper training error subject to the $\varepsilon$ insensitive tube $y_{i}-\left\langle w, x_{i}\right\rangle-b \leq \varepsilon$.The parameters which control the regression quality are the cost of error $C$, the width of the tube $\varepsilon$, and the mapping function $\phi$.

QPSO was inspired by analysis of the convergence of the traditional PSO and quantum system[5]. In the quantum physics, the state of a particle with momentum and energy can be depicted by its wave function $\Psi(x, t)$.

According to QPSO theory each particle is in a quantum state and is formulated by its wave function $\Psi(x, t)$ instead of the position and velocity which are in PSO[6]. According to the statistical significance of the wave function, the probability of a particle's appearing in a certain position can be obtained from the probability density function $|\Psi(x, t)|^{2}$.

And then the probability distribution function of the particle's position can be calculated through the probability density function. By employing the Monte Carlo method, the particle's position is updated according to the following equation:

$$
X_{i j}^{t+1}=p_{i j}^{t} \pm 0.5 * L_{i j}^{t} * \ln \left(\frac{1}{u_{i j}^{t}}\right)
$$

where ${ }^{t}{ }^{t}$ is a random number uniformly distributed in $(0,1) ; P_{i j}^{t}$ is the local attractor and defined as

$$
P_{i j}^{t}=\varphi_{i j}^{t} * P_{i j}^{t}+\left(1-\varphi_{i j}^{t}\right) * P_{g j}^{t}
$$

where $\varphi_{i j}^{t}$ is a random number uniformly distributed in $(0,1),{ }_{g j}^{t}$ is the global best position. In parameter $L_{i j}^{t}$ is evaluated by

$$
L_{i j}^{t}=2 * \beta *\left|P_{i j}^{t}-X_{i j}^{t}\right|
$$

where parameter $\beta$ is called the contraction-sxpansion(CE) coefficient, which can be tuned to control the convergence speed of the algorithms. Then we get the position update equation as

$$
X_{i j}^{t+1}=P_{i j}^{t} \pm \beta^{*}\left|P_{i j}^{t}-X_{i j}^{t}\right| * \ln \left(\frac{1}{U_{i j}^{t}}\right)
$$

The PSO algorithm with position update equation (5) is called as quantum delta-potential-well-based PSO (QDPSO) algorithm. Keeping in view the vital position of L for convergence rate and performance of the algorithm an improvement was proposed to evaluate parameter 1. As per this algorithm the mean best position (mbest) is defined as the center of pbest positions of the swarm. That is mbestt $=($ mbest $1 \mathrm{t}$, mbest $2 \mathrm{t}$, mbest $3 \mathrm{t} . . .$, mbest $\mathrm{Dt})$

$$
\frac{1}{M} \sum_{i=1}^{M} P_{i 1}^{t}, \frac{1}{M} \sum_{i=1}^{M} P_{i 2}^{t}, \ldots \frac{1}{M} \sum_{i=1}^{M} P_{i j}^{t}, \ldots \frac{1}{M} \sum_{i=1}^{M} P_{i D}^{t}
$$


The most commonly used control strategy of $b$ is to initially setting it to 1.0 and reducing it linearly to 0.5. In this study the normalized mean square error (NMSE) serves as the fitness criterion for identifying the suitable parameters for SVM model. The NMSE value of each particle is then determined using the fitness function:

$$
N M S E=\frac{n-1}{n} \frac{\sum_{i=1}^{n}\left[\left(Q_{m}\right)_{i}-\left(Q_{s}\right)_{i}\right]^{2}}{\sum_{i=1}^{n}\left[\left(Q_{m}\right)_{i}-\left(Q_{m}\right)_{i}\right]^{2}}
$$

where $\mathrm{Q}$ is the streamflow value and the subscripts ' $\mathrm{m}$ ' and ' $\mathrm{s}$ ' represent the measured and simulated values, respectively. The average value of associated variable is represented with a 'tilde' above it and $\mathrm{n}$ depicts the total number of training records.

\section{Experimental results}

Image encoding rules. The traceability system, the smallest unit of food for each generates a unique food code, the food encoding both a user query through landing pages to enter a code, while the coded encrypted data to generate two-dimensional code. Commodity code is automatically generated according to certain rules, for a total of 16, the structure shown in Fig. 3, two enterprise code +2 Category Code +48 Total production batch codes as classification and coding of goods, 8 random and repetitive code that uniquely identifies as a commodity.

For example: 0212010102211200

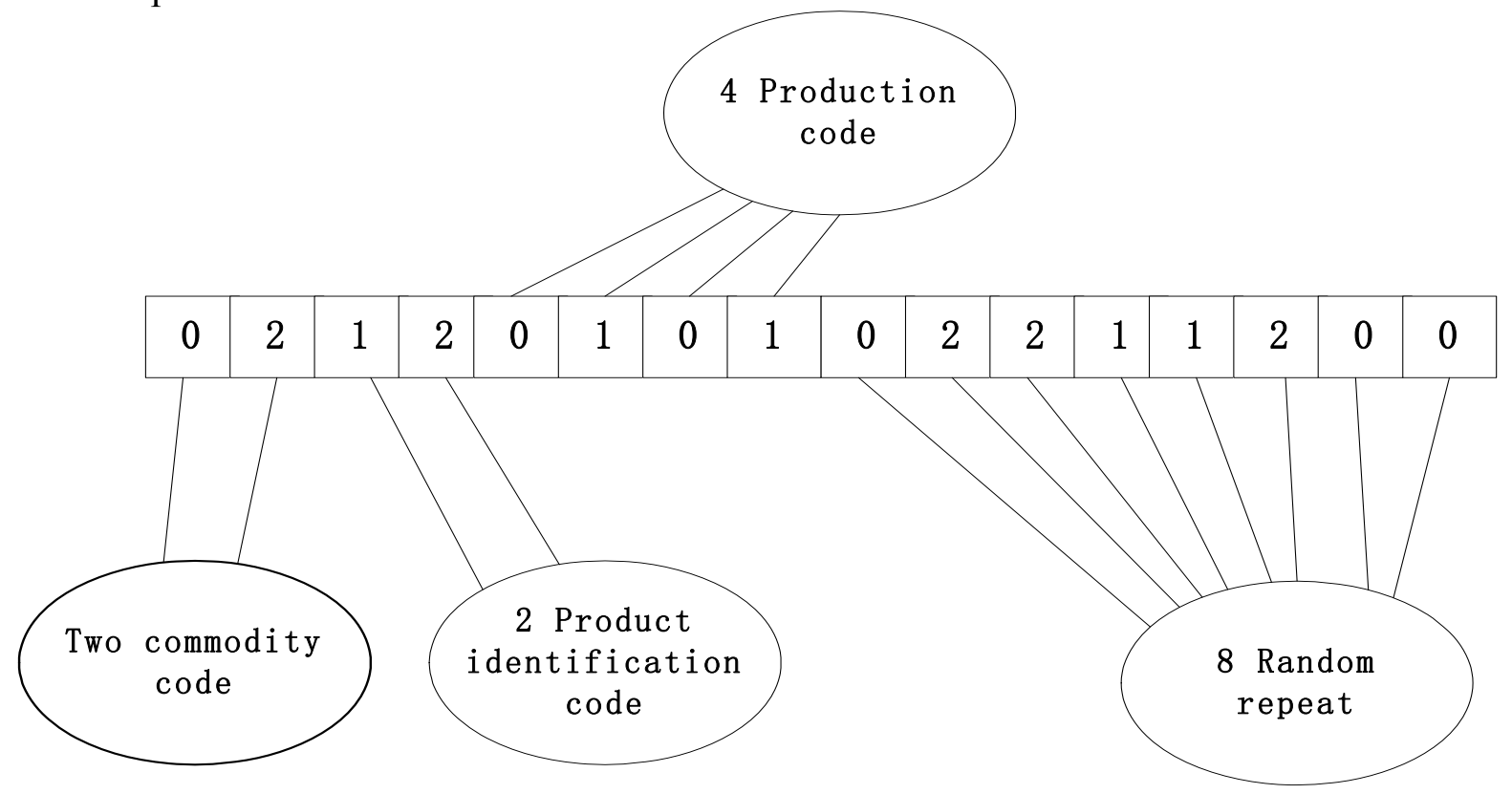

Fig. 3. The structure of QR code

Database management data support the most central role in food traceability system. Database system based on the logical relationship between the data to organize the data, the data provide storage, query, modify, and other related functions. A slightly superior database design is directly related to the availability of the use of the system, simplify the business logic play an effective role, but also effectively improve the performance of the system platform. Therefore, database design for food traceability system is particularly important, the system uses the relational model to represent database ER conceptual design, because the system data table more, following is a partial realization of the design of the database.

Simulation and Analysis. Logistics information collection by the enterprise logistics information manager, logistics enterprise information into the personnel, dealers or branch of the relevant staff to complete, each to a node, such persons by scanning software logistics code, recording the corresponding stream in the system information, form a closed-loop logistics information, providing accurate and timely information to businesses, consumers and other inquiries logistics information. Dimensional code recognition system stability monitoring image was shown in Fig. 4. 

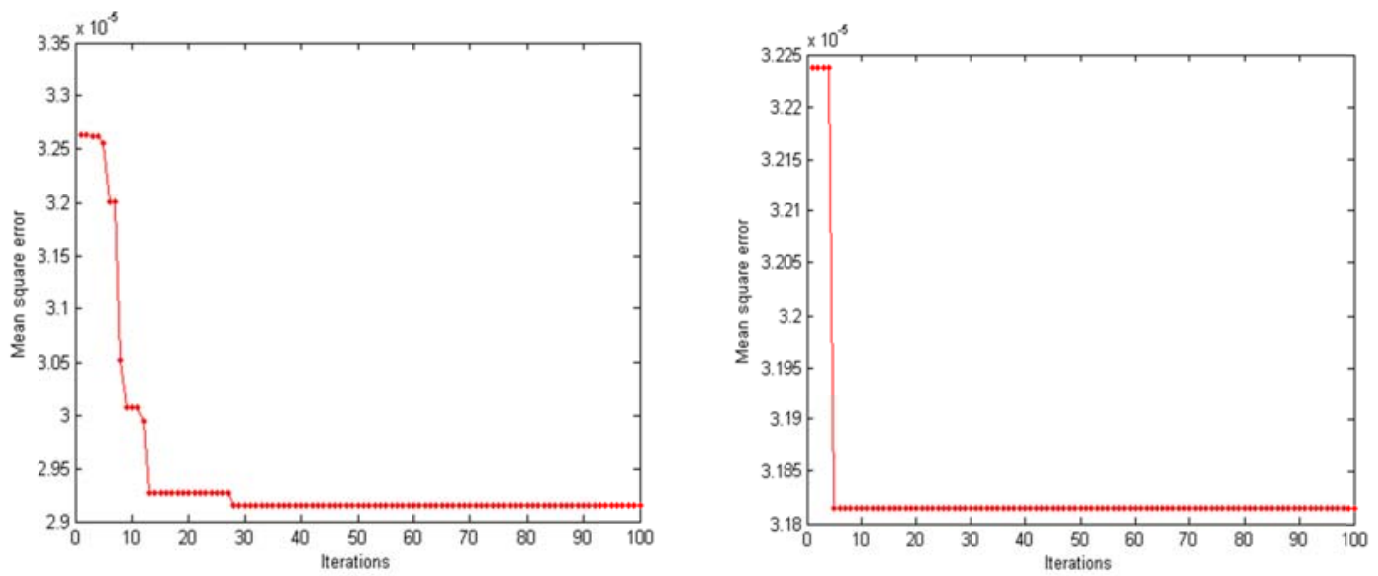

Fig. 4. Dimensional code recognition system stability monitoring image

\section{Summary}

The method has good quickness to meet the requirements of real-time detection and measurement accuracy is high. Through the above analysis shows that the improved stereo matching algorithms and image filtering method based on, can accurately reconstruct the original image, and with high precision, non-contact measurement, operational efficiency and many other advantages, it is very suitable for the logistics industry for security products, anti-string goods as well as for product tracking and other operations. In the image matching process, using partial matching based on adaptive weights, reducing the chance of mismatch, and further improve the accuracy and processing speed of the image. From the simulation results, the application of logistics management system that counterfeit detection method, the detection accuracy can meet the accuracy requirements of logistics security products detect identity.

\section{Acknowledgement}

This research was supported by Huaian Industry and Information Technology Program in 2015, supervised by Huai'an Municipal Science and Technology Bureau and Jiangsu Polytechnic of finance \&Economics.

\section{References}

[1] Hamad K, Chalhoub D. An RFID attendance and monitoringsystem for university application.Electronices, Circuits, andSystems(ICECS).2010: 851

[2] Hou, Yue, Mai, Yuemei. Chaotic prediction for traffic flow of improved BP neural networ. Telkomnika. 11, 3 (2012),pp. 1682-1690.

[3] Li Z, Yan X, Yuan C, Peng Z, Li L. Virtual prototype and experimental research gear multi-fault diagnosis using wavelet - autoregressive model and principal component analysis method. Mechanical Systems and Signal Processing. 25, 7 (2011),pp. 2589-2607.

[4] Sudheer, Ch; Anand, Nitin; Panigrahi, B. K. Streamflow forecasting by SVM with quantum behaved particle swarm optimization. NeuroComputing. 101, 2 (2013), pp. 18-23.

[5] Chen, Debao; Wang, Jiangtao; Zou, Feng. An improved group search optimizer with operation of quantum-behaved swarm and its application. Applied soft computing. 12,2(2012),pp. 712-725.

[6] Sudheer, Ch.; Shrivastava, Nitin Anand; Panigrahi, Bijaya Ketan. A Preliminary Estimate of Time and Cost in Urban Road Construction Using Neural Networks. Tehnicki Vjesnik-Technical Gazette. 3, 20(2013), pp. 563-570.

[7] A. Boldyreva. Threshold signature, multisignature and blind signature schemes based on the gap-Diffie-Hellmangroup signature scheme. In Proceedings of PKC 2003, LNCS 2567 , Springer, Berlin, 2003, pp.31-46. 